\section{CXCR4 upregulation is an indicator of sensitivity to B-cell receptor/PI3K blockade and a potential resistance mechanism in B-cell receptor-dependent diffuse large B-cell lymphomas}

\begin{abstract}
Linfeng Chen, ${ }^{1,2}$ Jing Ouyang, ${ }^{1 *}$ Kirsty Wienand, ${ }^{1 *}$ Kamil Bojarczuk,${ }^{1,3^{*}}$ Yansheng Hao, ${ }^{1,4}$ Bjoern Chapuy, ${ }^{1,5}$ Donna Neuberg, ${ }^{6}$ Przemyslaw Juszczynski, ${ }^{1,3}$ Lee N. Lawton, ${ }^{1}$ Scott J. Rodig, ${ }^{7}$ Stefano Monti $^{8}$ and Margaret A. Shipp ${ }^{1}$

${ }^{1}$ Department of Medical Oncology, Dana-Farber Cancer Institute, Boston, MA, USA; ${ }^{2}$ Current address: H3 Biomedicine, Cambridge, MA, USA; ${ }^{3}$ Current address: Department of Experimental Hematology, Institute of Hematology and Transfusion Medicine, Warsaw, Poland; ${ }^{4}$ Current Address: Department of Pathology, Mount Sinai Hospital, New York, NY, USA; ${ }^{5}$ Current Address: Department of Hematology and Oncology, University Medical Center Göttingen, Göttingen, Germany; ${ }^{6}$ Department of Biostatistics and Computational Biology, Dana-Farber Cancer Institute, Boston, USA; 'Department of Pathology, Brigham and Women's Hospital, Boston, MA, USA and 'Section of Computational Biomedicine, Boston University School of Medicine, Boston, MA, USA
\end{abstract}

*JO, KW and KB contributed equally to this work.
B -cell receptor (BCR) signaling pathway components represent promising treatment targets in multiple B-cell malignancies including diffuse large B-cell lymphoma (DLBCL). In in vitro and in vivo model systems, a subset of DLBCLs depend upon BCR survival signals and respond to proximal BCR/phosphoinositide 3 kinase (PI3K) blockade. However, singleagent BCR pathway inhibitors have had more limited activity in patients with DLBCL, underscoring the need for indicators of sensitivity to BCR blockade and insights into potential resistance mechanisms. Here, we report highly significant transcriptional upregulation of $\mathrm{C}-\mathrm{X}-\mathrm{C}$ chemokine receptor 4 (CXCR4) in BCR-dependent DLBCL cell lines and primary tumors following chemical spleen tyrosine kinase (SYK) inhibition, molecular SYK depletion or chemical PI3K blockade. SYK or PI3K inhibition also selectively upregulated cell surface CXCR4 protein expression in BCRdependent DLBCLs. CXCR4 expression was directly modulated by forkhead box $\mathrm{O} 1$ via the PI3K/protein kinase B/forkhead box O1 signaling axis. Following chemical SYK inhibition, all BCR-dependent DLBCLs exhibited significantly increased stromal cell-derived factor-1 $\alpha$ (SDF-1 $\alpha$ ) induced chemotaxis, consistent with the role of CXCR4 signaling in B-cell migration. Select PI3K isoform inhibitors also augmented SDF-1 $\alpha$ induced chemotaxis. These data define CXCR4 upregulation as an indicator of sensitivity to BCR/PI3K blockade and identify CXCR4 signaling as a potential resistance mechanism in BCR-dependent DLBCLs.

\section{Introduction}

Diffuse large-B-cell lymphomas (DLBCLs) are clinically and genetically heterogeneous diseases. ${ }^{1}$ Our previous studies demonstrated that a subset of DLBCLs rely upon B-cell receptor (BCR)-dependent survival signals. ${ }^{2,3}$ BCR signaling activates proximal pathway components including the spleen tyrosine kinase (SYK) and downstream effectors such as phosphatidylinositol-3-kinase (PI3K)/AKT and the Bruton's tyrosine kinase (BTK)/ nuclear factor- $\kappa \mathrm{B}$ (NF- $\kappa \mathrm{B}) \cdot{ }^{3,4}$ In prior studies, we, and others, characterized distinct BCR/PI3K-dependent viability pathways in DLBCL cell lines and primary tumors with low- or high-baseline NF-kB activity
Haematologica 2020

Volume 105(5):1361-1368

\section{Correspondence:}

MARGARET A. SHIPP

margaret_shipp@dfci.harvard.edu

Received: January 7, 2019.

Accepted: September 26, 2019.

Pre-published: October 3, 2019.

doi:10.3324/haematol.2019.216218

Check the online version for the most updated information on this article, online supplements, and information on authorship \& disclosures: www.haematologica.org/content/105/1361

(C)2020 Ferrata Storti Foundation

Material published in Haematologica is covered by copyright. All rights are reserved to the Ferrata Storti Foundation. Use of published material is allowed under the following terms and conditions:

https://creativecommons.org/licenses/by-nc/4.0/legalcode. Copies of published material are allowed for personal or internal use. Sharing published material for non-commercial purposes is subject to the following conditions:

https://creativecommons. org//icenses/by-nc/4.0/legalcode, sect. 3. Reproducing and sharing published material for commercial purposes is not allowed without permission in writing from the publisher. 
(germinal center B- (GCB-) and activated B-cell like (ABC)-type tumors, respectively). ${ }^{3,5-7}$

In both types of BCR-dependent DLBCLs, inhibition of SYK or PIBK decrease the phosphorylation of AKT and Forkhead Box O1 (FOXO1) and increase the nuclear retention and associated activity of unphosphorylated FOXO. ${ }^{13,8}$ BCR-dependent DLBCLs with low baseline NF-кB (GCB tumors) frequently exhibit inactivating mutations or copy loss of Phosphatase and tensin homolog (PTEN) and decreased abundance of the PTEN protein. ${ }^{1,3,6}$ In these DLBCLs, proximal inhibition of BCR signaling primarily modulates the PI3K/AKT pathway. ${ }^{3,5-}$ ${ }^{7,9}$ In contrast, SYK/PI3K blockade additionally limits BTK/NF- $\kappa$ B signaling in BCR-dependent DLBCLs with high baseline NF- $\kappa \mathrm{B}$ activity and frequent $M Y D 88^{\mathrm{L} 265 \mathrm{P}}$ and/or CD79B mutations (ABC tumors). ${ }^{1,3,7,9}$

We sought to identify an indicator of BCR dependence in DLBCLs with low or high baseline NF- $\kappa B$ and noted that C-X-C chemokine receptor 4 (CXCR4) transcripts were significantly more abundant in both DLBCL subtypes following the inhibition of proximal BCR signaling. ${ }^{3}$ In experimental model systems, BCR engagement promotes the internalization of CXCR4 and limits stromal cell-derived factor- $1 \alpha$ ) (SDF-1 $\alpha$ )-induced chemotaxis. ${ }^{10}$ For these reasons, we hypothesized that BCR blockade might increase CXCR4 expression and associated tumor cell migration.

Physiologically, the CXCR4 chemokine receptor binds to SDF-1 1 and plays a critical role in the chemotaxis of normal germinal center (GC) B cells. ${ }^{11-13}$ CXCR4 is a known FOXO1 target gene that is induced in normal FOXO1-rich dark zone GC B-cells. ${ }^{13}$ In the GC, CXCR4 ${ }^{+}$ $\mathrm{B}$-cells migrate in response to a SDF- $1 \alpha$ chemokine gradient. $^{11}$

CXCR4 transduces SDF-1 $\alpha$ signals via G-protein coupled activation of PIBK isoforms. ${ }^{14-18}$ As a consequence, CXCR4 is also considered to be a possible therapeutic target in multiple B-cell malignancies, including DLBCL. ${ }^{19-24}$ Herein, we assess CXCR4 modulation and signaling as both an indicator of sensitivity to BCR blockade and a potential resistance mechanism in DLBCL.

\section{Methods}

\section{Cell lines and culture conditions}

The DLBCL cell lines, SU-DHL4 (DHL4), SU-DHL6 (DHL6), OCI-LY7 (LY7), HBL1, TMD8, U-2932, Karpas 422 (K422), Toledo and OCI-LY4 (LY4), were cultured as previously described. ${ }^{25}$ The identities of the DLBCL cell lines used in this study were confirmed via STR profiling with PowerPlex ${ }^{\circledR} 1.2$ system (Promega, Madison, WI, USA). DHL4, DHL6, LY7, HBL1 and U-2932 were previously characterized as BCRdependent and K422, Toledo and LY4 were BCR-independent.,

\section{Primary tumor specimens}

Cryopreserved viable primary DLBCL samples were obtained according to the Institutional Review Board (IRB) - approved protocols from the Brigham and Women's Hospital Department of Pathology. These anonymous primary tumor specimens were considered discarded tissues which did not require informed consent. The six primary DLBCLs were previously characterized for surface immunoglobulin (Ig) expression, BCR signaling and baseline NF- $\kappa \mathrm{B}$ activity. ${ }^{3}$

\section{Chemical inhibition of SYK, PI3K or BTK}

The chemical SYK inhibitor, R406, was a gift from Rigel Pharmaceuticals (San Francisco, CA, USA). R406 was dissolved in DMSO at a concentration of $10 \mathrm{mM}$ and stored at $-80^{\circ} \mathrm{C}$. For immediate inhibition, cells were incubated with $1 \mu \mathrm{M}$ R406 or vehicle alone (in PBS) in a $37^{\circ} \mathrm{C}$ water bath for 2 hours (h). For long-term inhibition, R406 was added to cell culture medium at a final concentration of $1 \mu \mathrm{M}$ and cells were maintained in an incubator at $37^{\circ} \mathrm{C}$ for $24 \mathrm{~h}$. The chemical pan-PI3K inhibitor, LY294002, was purchased from Sigma-Aldrich (Saint Louis, MO, USA), The chemical SYK inhibitor, GS-9973 (entospletinib), the PI3K isoform-predominant inhibitors, GDC-0941 (pictilisib, PI3K $\alpha / \delta>\beta / \gamma$ ), CAL101 (idelalisib, $\delta$ ) and IPI145 (duvelisib, $\delta / \gamma$ ) and the BTK inhibitor, PCI-32765 (ibrutinib) were purchased from Selleckchem (Houston, TX, USA). DLBCL cell lines were treated with GS-9973 $(2 \mu \mathrm{M})$, LY294002 $(10 \mu \mathrm{M})$, GDC-0941 $(0.5 \mu \mathrm{M})$, CAL101 $(2 \mu \mathrm{M})$, IPI145 (1 $\mu \mathrm{M})$, PC1-32765 (0.1 $\mu \mathrm{M})$ or vehicle (DMSO) for $24 \mathrm{~h}$ as previously described. ${ }^{9}$ The doses of SYK, PI3K and BTK inhibitors used in these studies were determined based on prior analyses of the respective agent EC50s of these agents; ${ }^{9}$ the LY294002 dose was chosen based on previously reported studies. ${ }^{3,16}$ Following treatment with chemical SYK, PI3K or BTK inhibitors, cells were harvested for additional analyses (below).

\section{Quantitative RT-PCR (qRT-PCR)}

ORT-PCR was performed as previously described ${ }^{9}$ (Online Supplementary Materials and Methods).

\section{Flow cytometry}

A PE-conjugated mouse anti-human CD184 (CXCR4) antibody (BD Bioscience, CA, USA) was used for flow cytometry analysis on a FACS Canto II flow cytometer (BD Biosciences) and the data were analyzed with FlowJo 10 software (Flowjo Data analysis software LLC, Ashland, OR, USA).

\section{Lentiviral-mediated shRNA transduction}

The shRNA knockdown of target genes was performed as previously described ${ }^{3}$ (Online Supplementary Materials and Methods).

\section{Transduction with myristoylated AKT}

DLBCL cell lines were retrovirally transduced with constructs encoding constitutively active (myristoylated) AKT (pMIGmAKT1-IRES-GFP) or pMIG-IRES-GFP as previously described. ${ }^{3}$ After 72 h, $\mathrm{GFP}^{+}$cells were sorted, treated with R406 or vehicle, and analyzed for CXCR4 expression by flow cytometry.

\section{Chemotaxis assay}

DLBCL cell lines were treated with vehicle, R406 (1 $\mu \mathrm{M})$, GDC-0941 $(0.5 \mu \mathrm{M})$, Ibrutinib $(0.1 \mu \mathrm{M})$, AMD3100 $(10 \mu \mathrm{M})$ or R406 + AMD3100 for 24 h. Before the chemotaxis assay, Permeable Polycarbonate Membrane Inserts in the Corning ${ }^{\mathrm{TM}}$ Transwell ${ }^{\mathrm{TM}}$ 24-well plate (Fisher Scientific, pore size $8 \mu \mathrm{m}$ ) were pretreated by adding $600 \mu \mathrm{L}$ of RPMI-1640 media containing $0.5 \%$ bovine serum albumin (Sigma) with or without SDF-1 $\alpha$ (25-100 ng/mL, R\&D Systems) into the bottom chamber at $37^{\circ} \mathrm{C}$ for $1 \mathrm{~h}$. Each lot of SDF-1 $\alpha$ was individually titrated for activity in the chemotaxis assay prior to use. Treated cells were harvested and resuspended in RPMI-1640 media for a final density of $2 \times 10^{6} / \mathrm{mL} .100 \mu \mathrm{L}$ of cell suspension was transferred to each top chamber and incubated at $37^{\circ} \mathrm{C}$ for $2-4 \mathrm{~h}$. Cells in the lower chambers were harvested and cell numbers were determined by manual counting. Each condition was set up in triplicate. 


\section{Results}

SYK inhibition selectively induces CXCR4 expression in BCR-dependent DLBCL cell lines and primary tumors

To identify potential compensatory signaling pathways in DLBCL treated with chemical BCR inhibitors, we reviewed the transcriptional profiles of five BCR-dependent DLBCL cell lines treated with the chemical SYK inhibitor, R406, or vehicle (DMSO). ${ }^{3}$ Differential analysis of treated versus untreated samples revealed that CXCR4 transcripts were significantly upregulated in all five BCR- dependent DLBCL cell lines (DHL4, DHL6, LY7, HBL1, U2932) following 6-24 h of R406 treatment $(P$-value $=0.00052$ at $24 \mathrm{~h}$; Online Supplementary Figure S1).

To expand on these findings, we treated an extended panel of BCR-dependent and BCR-independent DLBCL cell lines with R406 (or vehicle) and evaluated CXCR4 transcript abundance by qRT-PCR. The extended DLBCL cell line panel included five BCR-dependent DLBCL cell lines (DHL4, DHL6, LY7 [low NF-kB, GCB]; and HBL1 and U-2932 [high NF- $\mathrm{KB}, \mathrm{ABC}$ ]) ${ }^{3}$ and an additional $\mathrm{ABC}$ DLBCL cell line, TMD8, that is $\operatorname{sgM}^{+}, \mathrm{BCR}$-dependent,
A

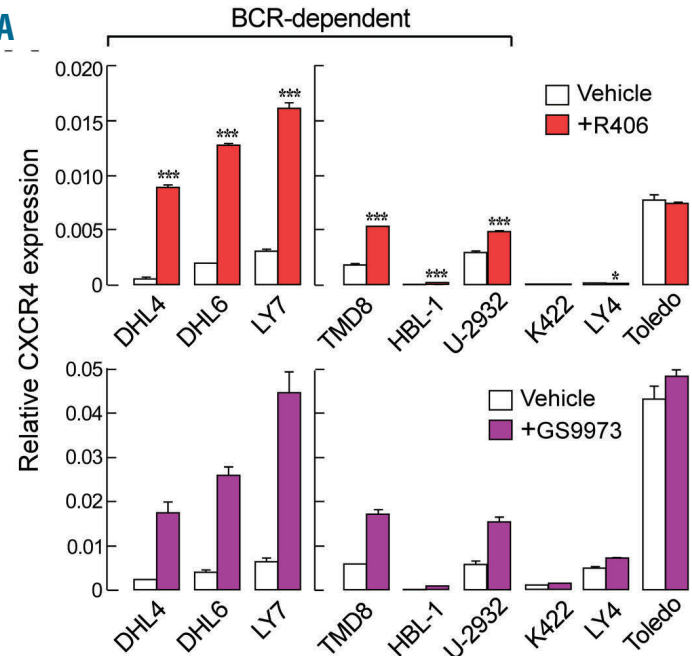

B

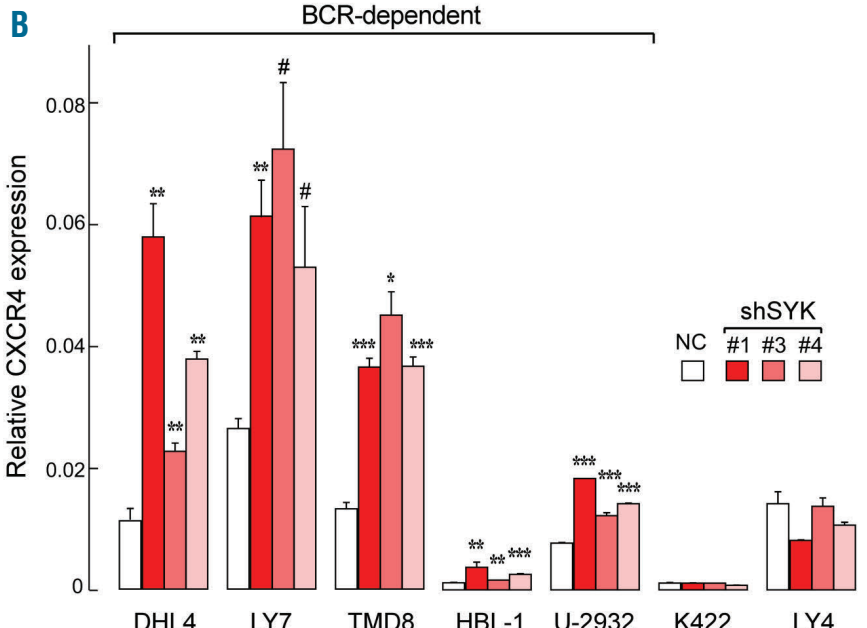

C

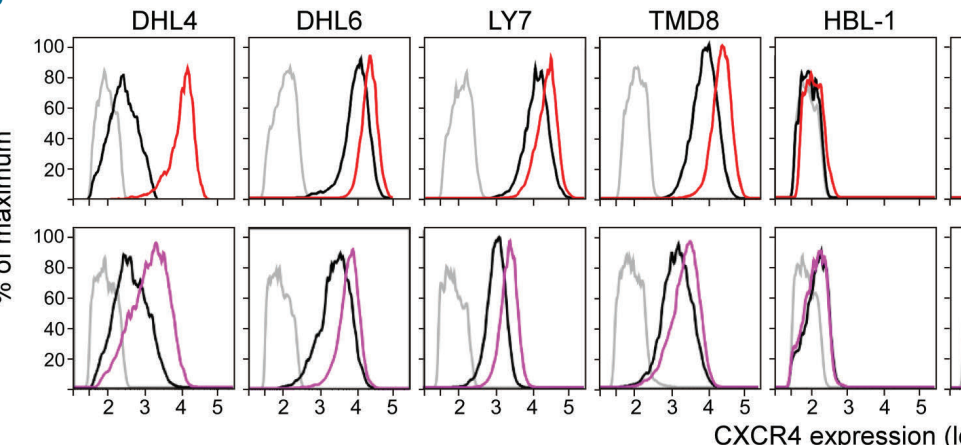

U-2932
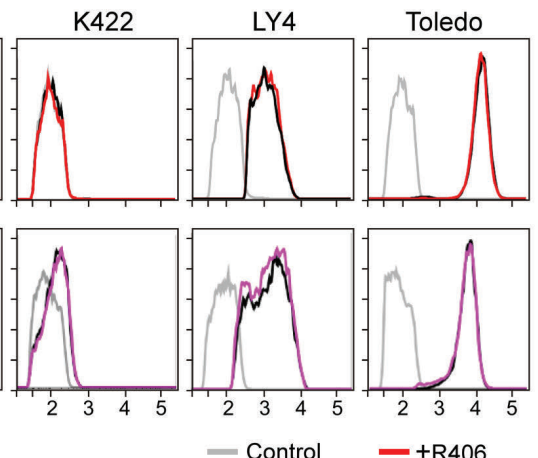

D

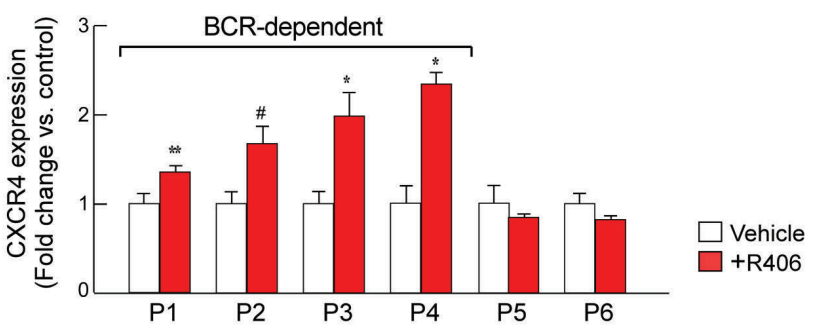

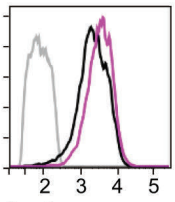

$\log )$
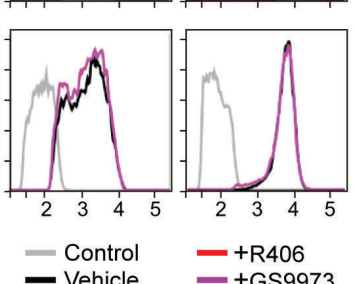

Figure 1. CXCR4 is upregulated in BCR-dependent DLBCL cell lines following SYK inhibition. (A) CXCR4 transcript abundance in diffuse large B-cell lymphoma (DLBCL) cell lines treated with 1 uM R406 (upper panel) or $2 \mu \mathrm{M}$ GS-9973 (lower panel) for 24 hours (h) was determined by quantitative RT-PCR (qRT-PCR) relative to PPIA. The P-values for vehicle versus R406 treated or GS-9973 treated were determined with a one-sided Welch $t$-test. $* * * P<0.0001$; $* P<0.01$. Error bars represent the SD of three independent assays in a representative experiment. (B) CXCR4 transcript abundance in SYK-depleted DLBCL cell lines (72 h following completion of puromycin selection) was determined by qRT-PCR relative to PPIA. NC (negative control) shRNA. The $P$-values for NC versus shSYK constructs were determined with a one-sided Welch $t$-test. $* * * P<0.0001 ; * * P<0.001 ; * P<0.01 ;{ }^{*} P<0.05$. Error bars represent the SD of three independent assays in a representative experiment. (C) Cell surface expression of CXCR4 in DLBCL cell lines treated for $24 \mathrm{~h}$ with vehicle or $1 \mu \mathrm{M}$ R406 (upper panel), or vehicle or $2 \mu \mathrm{M}$ GS-9973 (lower panel) was measured by flow cytometry. Isotype-matched control in gray. (D) CXCR4 expression in primary DLBCL patient samples following SYK inhibition. Cryopreserved viable DLBCL tumor cell suspensions from newly diagnosed patients were thawed and treated with vehicle or $1 \mu \mathrm{M}$ R406 for $24 \mathrm{~h}$. RNA samples were prepared and CXCR4 expression was determined by qRT-PCR relative to PPIA. The P-values for vehicle versus R406 treatment were determined with a one-sided Welch t-test. ${ }^{*} P<0.001 ; * P<0.01 ;{ }^{*} P<0.05$. Error bars represent the SD of three independent assays in a representative experiment. 
and sensitive to chemical SYK inhibition (R406) and molecular depletion of SYK (Online Supplementary Figure S2). Consistent with its designation as an ABC-type DLBCL cell line, TMD8 exhibited high baseline expression of the NF- $\kappa \mathrm{B}$ target, BCL2A1, that was markedly reduced following SYK depletion (Online Supplementary Figure S2). The extended cell line panel also included three BCR-independent DLBCL cell lines, K422, Toledo and LY4. ${ }^{3}$

In the DLBCL cell line panel, chemical SYK inhibition with R406 selectively increased CXCR4 transcript levels in all six BCR-dependent DLBCL cell lines; however, the baseline CXCR4 levels in HBL1 were low. Chemical SYK inhibition did not modulate CXCR4 transcript abundance in the three BCR-independent DLBCL cell lines (Figure 1A, top panel). Similar results were obtained with a more selective chemical SYK inhibitor, GS-9973, that is currently under evaluation in lymphoma clinical trials (Figure 1A, lower panel). ${ }^{26-28}$

SYK depletion with three independent shRNAs significantly increased CXCR4 transcripts in BCR-dependent, but not BCR-independent DLBCL cell lines, phenocopying the CXCR4 induction following chemical SYK inhibition (Figure 1B). Consistent with these findings, chemical inhibition of SYK with either R406 or GS-9973 selectively increased cell surface CXCR4 protein expression in the BCR-dependent DLBCLs (with the least effect in HBL1), but not in the BCR-independent DLBCLs (Figure 1C, R406, top panel; GS-9973, bottom panel).

After identifying selective CXCR4 induction in BCRdependent DLBCLs cell lines, we assessed the same parameters in primary DLBCLs. For these studies, we uti- lized aliquots of six cryopreserved viable tumor suspensions of primary DLBCL that were previously characterized as BCR-dependent with low baseline NF- $\mathrm{B}$ activity (P1 and P2), BCR-dependent with high baseline NF- $\mathrm{B}$ activity (P3 and P4); or BCR-independent (P5 and P6). ${ }^{3}$ As in the DLBCL cell lines, chemical SYK inhibition selectively induced CXCR4 in all four BCR-dependent primary DLBCLs (P1-P4) but not in the two BCR-independent primary DLBCLs (P5 and P6) (Figure 1D).

\section{Prolonged chemical SYK inhibition increases SDF-1 $\alpha$ associated migration of BCR-dependent DLBCLs}

We next assessed the functional significance of CXCR4 induction following prolonged SYK inhibition by performing a transwell chemotaxis assay using SDF-1 $\alpha$ as the chemoattractant. Prolonged SYK blockade selectively enhanced the migration of all examined BCR-dependent DLBCL cell lines to SDF-1 $\alpha$; the migration of the BCRindependent DLBCL cell lines was unchanged (Figure 2A). The R406-augmented, SDF-1 $\alpha$ associated cellular migration was abrogated when the chemotaxis assay was performed in the presence of the specific CXCR4 inhibitor AMD3100, confirming the specificity of the observed effect (Figure 2B).

\section{PI3K/AKT signaling regulates CXCR4 expression in BCR-dependent DLBCL cell lines}

We previously described the central role of PI3K/AKT in SYK-mediated BCR-signaling in DLBCLs. ${ }^{3,9}$ These data prompted us to evaluate the function of PI3K/AKT in the regulation of CXCR4 upon proximal BCR/PI3K inhibition. For these studies, representative BCR-dependent DLBCL

A

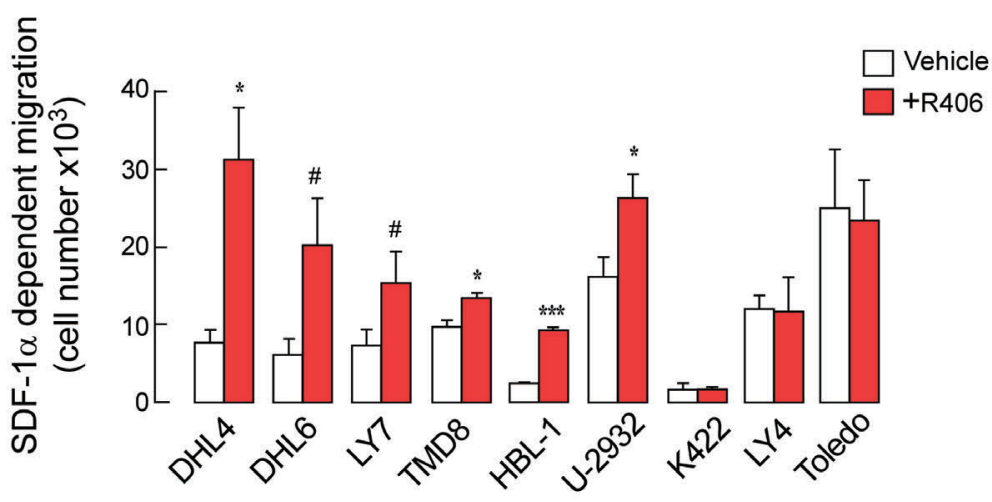

B

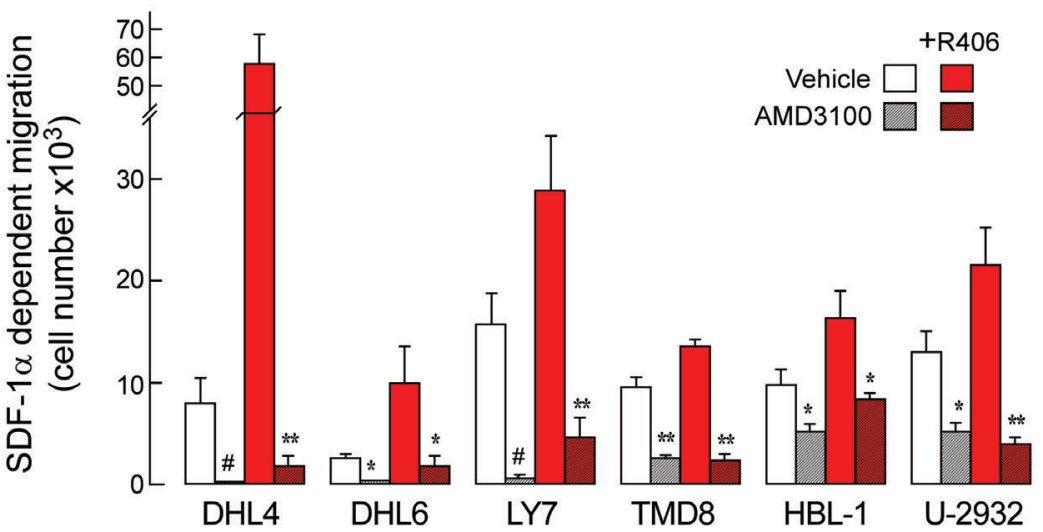

Figure 2. SDF-1 $\alpha$ induced cell migration in DLBCL cell lines following SYK inhibition. (A) DLBCL cells were treated with vehicle or R406 for 24 hours $(\mathrm{h})$, then assayed for migration in response to $100 \mathrm{ng} / \mathrm{mL} \mathrm{SDF}-1 \alpha$ for $4 \mathrm{~h}\left(2 \times 10^{5}\right.$ cells per condition). (B) BCR-dependent DLBCL cell lines were treated with vehicle, $10 \mu \mathrm{M}$ AMD3100, 1 uM R406 or combination of AMD3100 and R406 for $24 \mathrm{~h}$, then assayed for migration in response to SDF- $1 \alpha$. The $P$-values for vehicle- versus R406-treated (A) and vehicle- versus AMD3100-treated or R406 alone versus AMD3100+R406 (B) were determined with a one-sided Welch $t$-test. $* * * P<0.0001$; $* * P<0.001 ; * P<0.01 ;{ }^{*} P<0.05$. Error bars represent the SD of three independent assays in a representative experiment. 
cell lines (DHL4, DHL6, LY7 [low NF-кB]; and TMD8 [high NF-кB]), were transduced with a vector encoding constitutively active (myristoylated) AKT1 (mAKT) or an empty vector control. ${ }^{29}$ Thereafter, $\mathrm{GFP}^{+}$-selected cells were treated with vehicle control or R406 and analyzed for CXCR4 expression. Following R406 treatment, all four BCR-dependent DLBCL cell lines infected with the control vector expressed increased CXCR4 (Figure 3A, top panel). In contrast, chemical SYK inhibition did not modulate CXCR4 expression in mAKT-expressing DLBCL cell lines with constitutive activation of AKT1 (Figure 3A, lower panel). These data confirmed the role of PIBK/AKT in
SYK-dependent modulation of CXCR4 expression.

Given these findings, we assessed the consequences of chemical pan-PI3K inhibition on CXCR4 expression in BCR-dependent DLBCL cell lines (Figure 3B) using the tool compound, LY294002. Like chemical SYK inhibition, panPI3K blockade with LY294002 increased CXCR4 transcript abundance (Figure $3 \mathrm{~B}$ ) and cell surface expression (Figure $3 C)$.

We next examined the mechanism by which prolonged SYK/PI3K inhibition induces CXCR4 expression in BCRdependent DLBCLs. BCR signaling is known to promote CXCR4 internalization and inhibit SDF-1 $\alpha$ induced
A - Control - Vehicle -+ R406

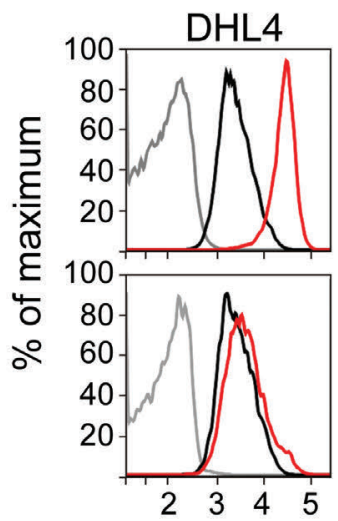

B
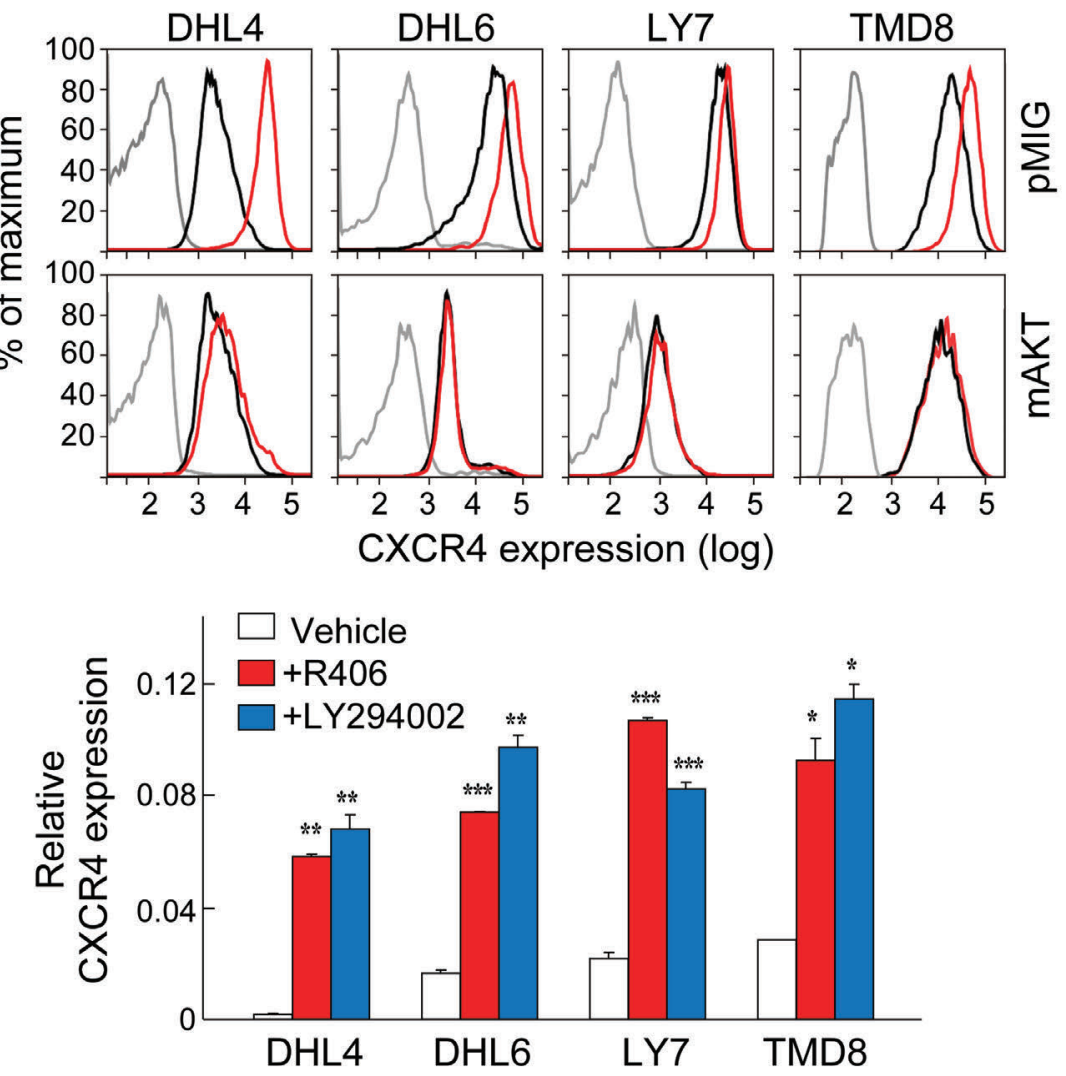

C _ Control - Vehicle - +R406 - +LY294002

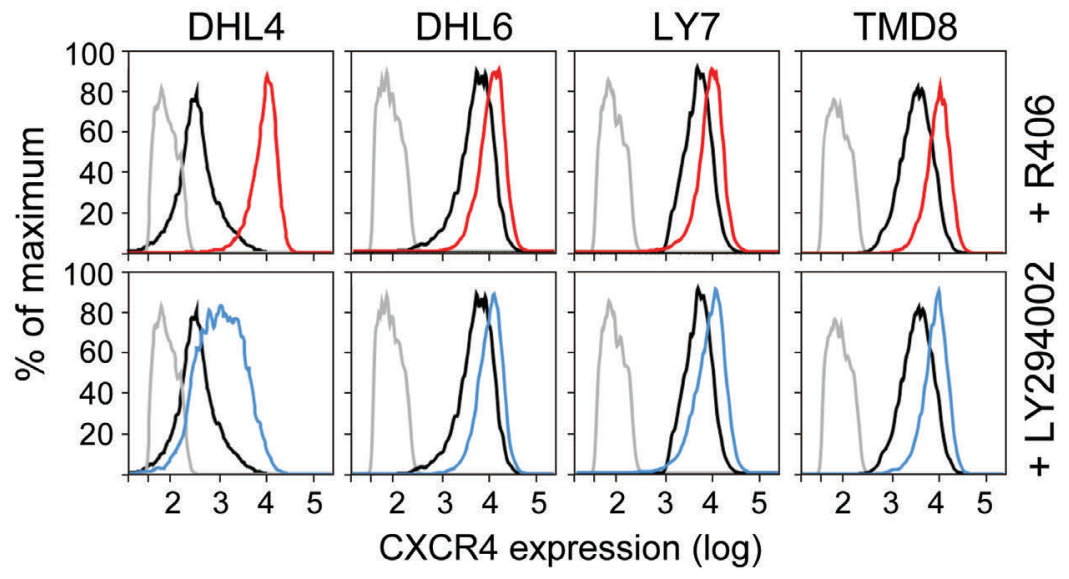

Figure 3. PI3K/AKT signaling regulates CXCR4 expression in BCR-dependent DLBCL cell lines. (A) BCR-dependent DLBCL cell lines, DHL4, DHL6, LY7 and TMD8, were retrovirally transduced with PMIGmAKT1-IRES-GFP or pMIG-IRES-GFP vector, FACSsorted for GFP expression, treated with $1 \mathrm{uM}$ R406 or vehicle for $24 \mathrm{~h}(\mathrm{~h})$, and analyzed for CXCR4 expression by flow cytometry. (B) BCR-dependent DLBCL cell lines were treated with $1 \mu \mathrm{M}$ R406 (red), $10 \mu$ M LY294002 (blue) or vehicle for $24 \mathrm{~h}$. Thereafter, CXCR4 expression was analyzed by qRT$\mathrm{PCR}$ relative to PPIA. The $P$-values for vehicle versus R406 treated or vehicle versus LY294002 treated were determined with a one-sided Welch t-test. $* * * P<0.0001 ; * * P<0.001 ; * P<0.01$. Error bars represent the SD of three independent assays in a representative experiment. (C) Cell surface expression of CXCR4 was measured by flow cytometry in DLBCL cell lines treated with vehicle (black), R406 (red) or LY294002 (blue) for $24 \mathrm{~h}$. 
chemotaxis. ${ }^{10}$ Therefore, molecular depletion or chemical inhibition of SYK or pan-PI3K blockade may limit CXCR4 internalization and increase residual cell surface CXCR4 expression. However, SYK/PI3K inhibition also increases nuclear localization of FOXO1 and associated FOXO1mediated transactivation of CXCR4 $4^{3,8,13,30}$ For these reasons, we depleted FOXO1 in a BCR-dependent DLBCL cell line (DHL4), treated the cells with vehicle or R406 and subsequently measured CXCR4 expression by flow cytometry (Online Supplementary Figure S3). SYK inhibition induced less CXCR4 in FOXO1-depleted cells (Online Supplementary Figure S3), highlighting the role of FOXO1 in CXCR4 expression.
After demonstrating CXCR4 upregulation following SYK or pan-PI3K inhibition (Figure 1 and Figure 3), we assessed the consequences of more selective PI3K isoform or BTK blockade using the $\mathrm{PI} 3 \mathrm{~K} \alpha / \delta>\beta / \gamma$, PI3K $\delta$ and $\mathrm{PI} 3 \mathrm{~K} \delta / \gamma$ predominant inhibitors, GDC-0941, CAL101 and IPI145, respectively, and the BTK inhibitor, PCI-32765 (ibrutinib) ${ }^{9}$ (Figure 4A). In each of the evaluated BCRdependent DLBCL cell lines (DHL4, DHL6 and TMD8), more selective PI3K isoform inhibition with GDC-0941, CAL101 or IPI145 increased CXCR4 transcript abundance (Figure 4A) and CXCR4 cell surface protein expression (Figure 4B). In contrast, BTK blockade had more modest effects on CXCR4 transcript and protein expression in the

A

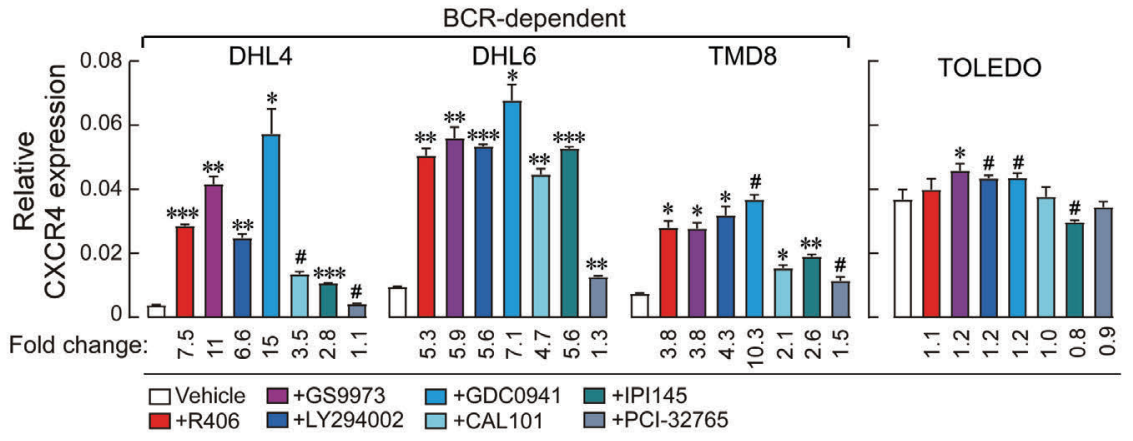

B

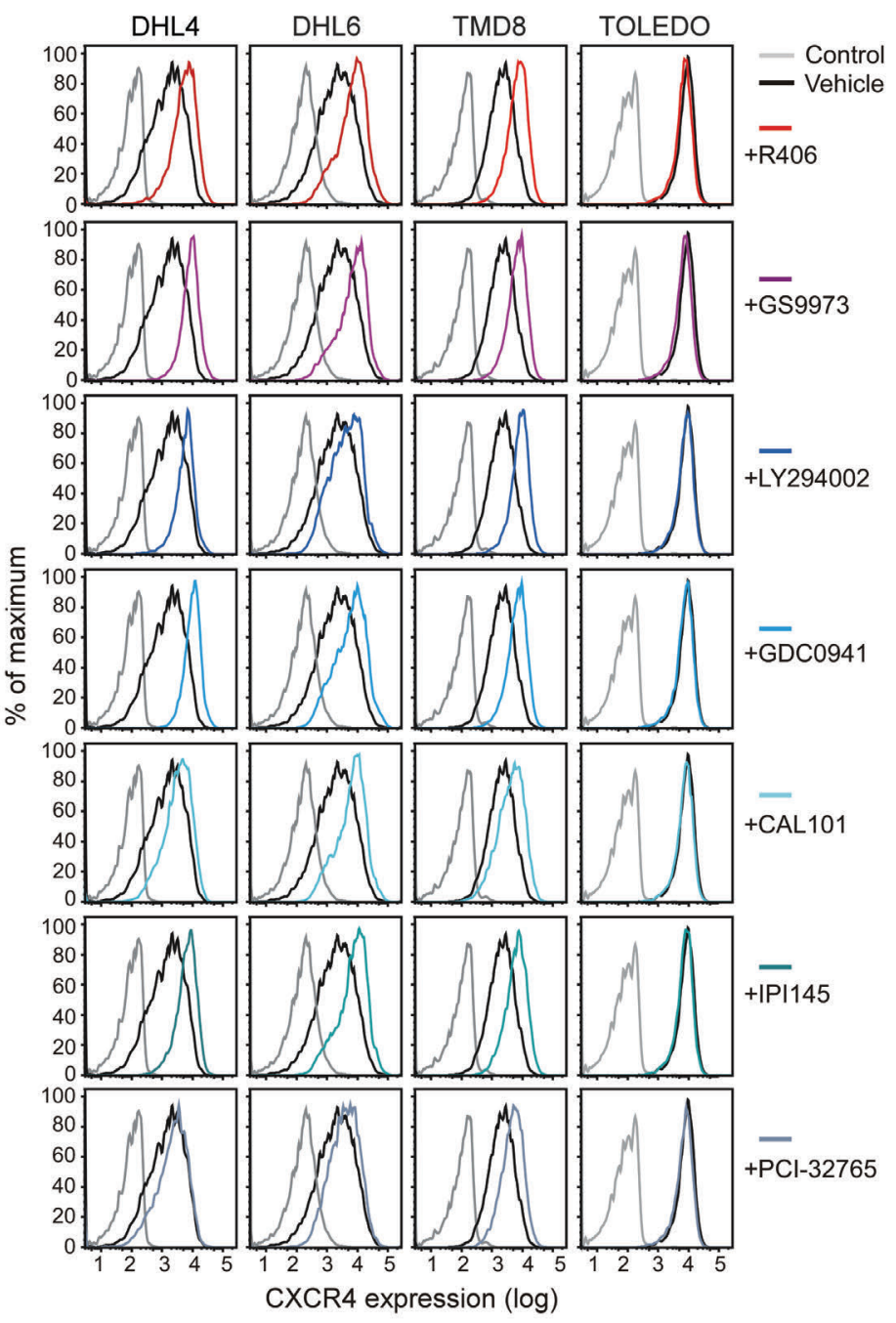

Figure 4. CXCR4 expression in BCR-dependent and BCR-independent DLBCL cell lines following SYK, PI3K or BTK inhibition. BCR-dependent diffuse large B-cell lymphoma (DLBCL) cell lines (DHL4, DHL6 and TMD8) and a BCR-independent DLBCL cell line (Toledo) were treated with DMSO, R406 (1 uM), GS-9973 (2 uM) LY294002 (10 $\mu \mathrm{M})$, GDC-0941 (0.5 $\mu \mathrm{M})$ CAL101 $(2 \mu \mathrm{M})$, IPI145 (1 $\mu \mathrm{M})$, PCI-32765 (0.1 $\mu \mathrm{M})$ for 24 hours (h). Thereafter, CXCR4 expression was analyzed by qRT-PCR relative to PPIA (A) or flow cytometry $(B)$ as in Figure $1 \mathrm{~A}$ and Figure 1C. (A) CXCR4 transcript abundance. Fold changes in CXCR4 transcript abundance relative to DMSO are shown below each inhibitor for the four cell lines. The $P$-values for vehicle versus R406, GS-9973, LY294002, GDC-0941, CAL101, IPI145 and PCl-32765 treated were determined with a one-sided Welch t-test. $* * * P<0.0001 ; \quad * * P<0.001$; ${ }^{\star} P<0.01 ;{ }^{*} P<0.05$. Error bars represent the SD of three independent assays in a representative experiment. (B) CXCR4 cell surface expression in DLBCL cell lines treated with vehicle (black) or the above-mentioned inhibitors (see key) for $24 \mathrm{~h}$. Isotype-matched control in gray. 


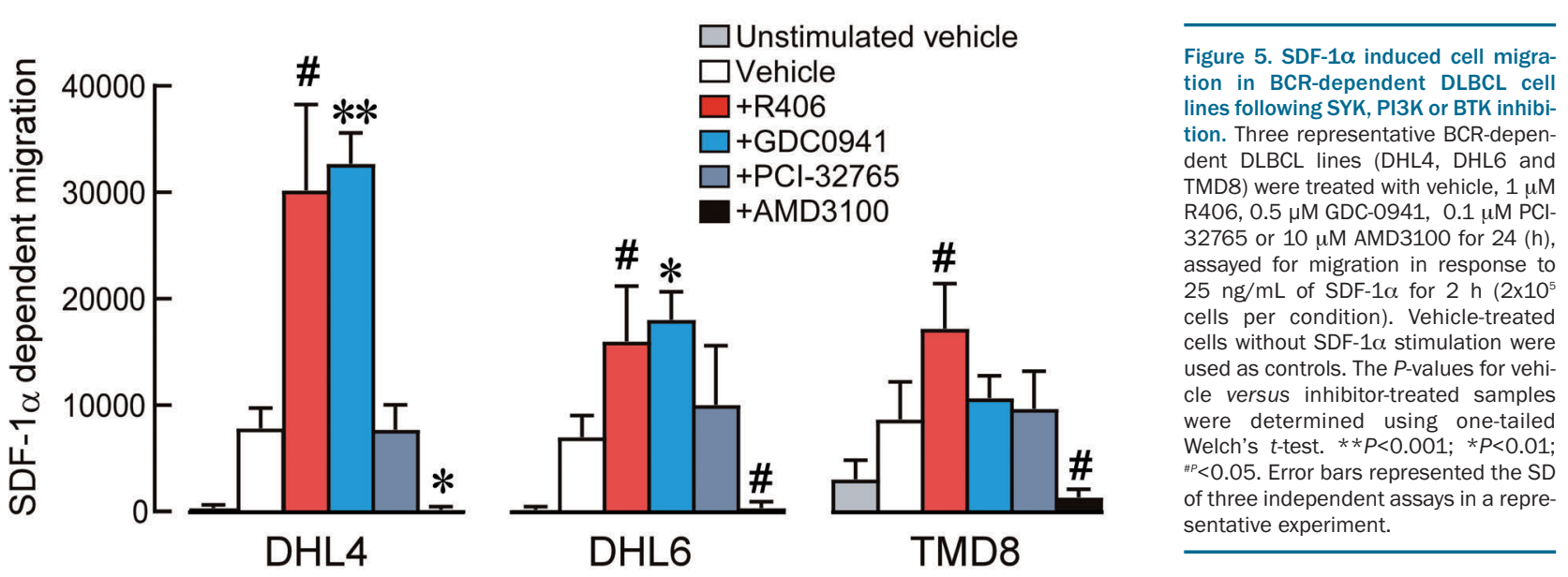

BCR-dependent lines. As expected, none of the compounds modulated CXCR4 expression in a BCR-independent DLBCL cell line (Toledo) (Figure 4A-B).

Consistent with these observations, chemical inhibition of SYK/PI3K was more effective than BTK blockade in augmenting SDF-1 $\alpha$ - induced chemotaxis (Figure 5).

\section{Discussion}

In this study, we identify CXCR4 upregulation as an indicator of sensitivity to targeted inhibition of BCR/PI3K signaling in DLBCL cell lines and primary tumor cell suspensions. Chemical SYK inhibition, genetic SYK depletion and PI3K inhibition all increased CXCR4 expression in BCRdependent DLBCLs. In DLBCLs with low or high baseline NF- $\kappa$ B, CXCR4 expression was modulated in a PI3K/AKT/FOXO1-dependent manner at the level of transcription (Figure 6). In addition to enhanced CXCR4 expression, proximal BCR(SYK)/PI3K inhibition induced chemotaxis of DLBCL cell lines to the CXCR4 ligand, SDF-1 $\alpha$.

In the current studies, we find induction of CXCR4 at the transcript level within 6 h of proximal BCR signaling blockade. Thereafter, increased CXCR4 cell surface expression is readily detectable by flow cytometry within $24 \mathrm{~h}$ of SYK or PI3K inhibition in almost all BCR-dependent DLBCLs. In recent studies, PI3K/mammalian target of rapamycin chemical inhibition also increased CXCR4 transcript abundance in BCR-dependent DLBCL cell lines. ${ }^{31}$ Taken together, these data suggest that CXCR4 upregulation is an indicator of sensitivity to inhibition of proximal BCR/PI3K signaling in DLBCLs.

CXCR4 is a FOXO1 target gene which, under physiological conditions, contributes to the polarization of light zone and dark zone GC-B cells. ${ }^{13}$ Upon inhibition of the BCR/SYK/PI3K/AKT axis, FOXO1 is dephosphorylated and retained in the nucleus, initiating transcription of its target genes. ${ }^{8} \mathrm{FOXO} 1$ is considered to be a homeostatic regulator with targets that include pro-apoptotic mediators of cell death such as BIM, HRK or p27, as well as BCR/PI3K signaling pathway components including SYK, PIK3CA and CXCR4 ${ }^{1,3,13}$ Therefore, FOXO1-dependent upregulation of CXCR4 can be regarded as a potential compensatory signaling pathway in DLBCLs following proximal BCR/PI3K inhibition.

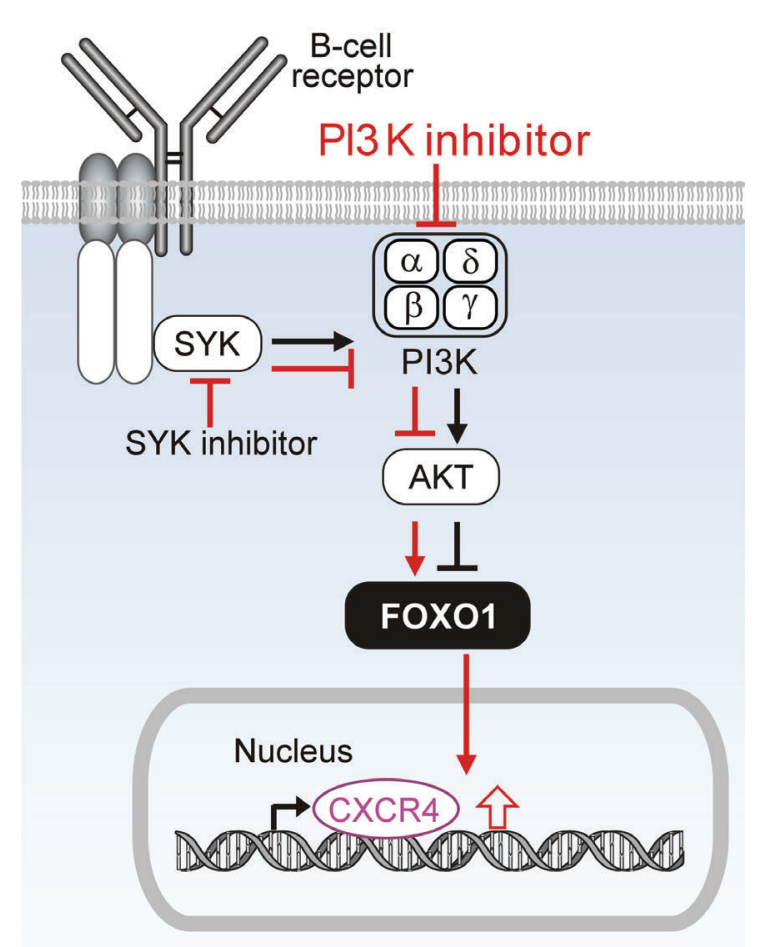

Figure 6. Model for B-cell receptor/SYK/PI3K regulation of CXCR4 signals. Red arrows indicate consequences of spleen tyrosine kinase (SYK) and/or phosphatidylinositol-3-kinase (PI3K) inhibition including increased nuclear localization of FOXO1 and C-X-C chemokine receptor 4 (CXCR4) upregulation.

In Waldenström's Macroglobulinemia (WM), nearly 30\% of patients exhibit an activating somatic mutation of CXCR $4^{32}$ that increases AKT and extracellular signal-regulated kinases signaling and mediates increased migration, adhesion, survival and resistance to ibrutinib. ${ }^{24}$ In our in vitro analyses of BCR-dependent DLBCLs, inhibition of SYK or PI3K signaling was more effective than BTK blockade in upregulating CXCR4 expression. In our recent genomic characterization of 304 primary DLBCLs, we did not observe recurrent CXCR4 mutations. ${ }^{1}$ However, immunohistochemical assessment of clinically annotated cohorts of de novo DLBCLs identified heterogeneity of CXCR4 expres- 
sion and adverse prognostic significance of CXCR4 staining. 33,34

These observations are noteworthy because CXCR4 may also be a relevant treatment target. There are ongoing clinical trials incorporating either the CXCR4 inhibitor (AMD3100, plerixafor) or a monoclonal antibody against CXCR4 (ulocuplumab) into existing therapies of WM. ${ }^{35}$ Additionally, multiple CXCR4 antagonists are reported to enhance the cytotoxic effect of rituximab or additional agents in diverse in vitro lymphoma models, including those of DLBCLs. ${ }^{19-23,36}$

Taken together, these data identify CXCR4 upregulation as an indicator of sensitivity to proximal BCR/PI3K blockade. These findings will potentially aid in the development of representative model systems and analyses of BCR/PI3K pathway-specific inhibitors. CXCR4 upregulation may also be an important and potentially targetable resistance mechanism in BCR-dependent DLBCLs.

\section{Funding}

This work was supported by a 'Mobility Plus' fellowship from the Polish Ministry of Science and Higher Education (1261/MOB/IV/2015/0) (KB) and a Leukemia and Lymphoma Society SCOR award (MA. and SJR).

\section{References}

1. Chapuy B, Stewart C, Dunford AJ, et al. Molecular subtypes of diffuse large B cell lymphoma are associated with distinct pathogenic mechanisms and outcomes. Nat Med. 2018;24(5):679-690.

2. Chen L, Monti S, Juszczynski P, et al. SYKdependent tonic B-cell receptor signaling is a rational treatment target in diffuse large B-cell lymphoma. Blood. 2008;111(4):2230-2237.

3. Chen L, Monti S, Juszczynski P, et al. SYK inhibition modulates distinct PI3K/AKTdependent survival pathways and cholesterol biosynthesis in diffuse large $B$ cell lymphomas. Cancer Cell. 2013;23(6):826-838.

4. Bojarczuk K, Bobrowicz M, Dwojak M, et al. $B$-cell receptor signaling in the pathogenesis of lymphoid malignancies. Blood Cells Mol Dis. 2015;55(3):255-265.

5. Havranek O, Xu J, Kohrer S, et al. Tonic B-cell receptor signaling in diffuse large B-cell lymphoma. Blood. 2017;130(8):995-1006.

6. Pfeifer M, Grau M, Lenze D, et al. PTEN loss defines a PI3K/AKT pathway-dependent germinal center subtype of diffuse large B-cell lymphoma. Proc Natl Acad Sci U S A. 2013;110(30):12420-12425.

7. Erdmann T, Klener P, Lynch JT, et al. Sensitivity to PIBK and AKT inhibitors is mediated by divergent molecular mechanisms in subtypes of DLBCL. Blood. 2017; 130(3):310-322

8. Szydlowski M, Kiliszek P, Sewastianik T, et al. FOXO1 activation is an effector of SYK and AKT inhibition in tonic BCR signaldependent diffuse large B-cell lymphomas. Blood. 2016;127(6):739-748.

9. Bojarczuk K, Wienand K, Ryan JA, et al. Targeted inhibition of PI3Kalpha/delta is synergistic with BCL-2 blockade in genetically defined subtypes of DLBCL. Blood. 2019;133(1):70-80.

10. Guinamard R, Signoret N, Ishiai M, Marsh M, Kurosaki T, Ravetch JV. B cell antigen receptor engagement inhibits stromal cellderived factor (SDF)-1alpha chemotaxis and promotes protein kinase C (PKC)-induced internalization of CXCR4. J Exp Med. 1999;189(9):1461-1466.

11. Allen CD, Ansel KM, Low C, et al. Germinal center dark and light zone organization is mediated by CXCR4 and CXCR5. Nat Immunol. 2004;5:943-952.

12. Victora GD, Dominguez-Sola D, Holmes AB, Deroubaix S, Dalla-Favera R, Nussenzweig $\mathrm{MC}$. Identification of human germinal center light and dark zone cells and their relationship to human B-cell lymphomas. Blood. 2012;120(11):2240-2248.

13. Dominguez-Sola D, Kung J, Holmes AB, et al. The FOXO1 Transcription factor instructs the germinal center dark zone Program. Immunity. 2015;43(6):1064-1074.

14. Thorpe LM, Yuzugullu H, Zhao JJ. PI3K in cancer: divergent roles of isoforms, modes of activation and therapeutic targeting. Nat Rev Cancer. 2015;15(1):7-24.

15. Janas ML, Varano G, Gudmundsson K, Noda M, Nagasawa T, Turner M. Thymic development beyond beta-selection requires phosphatidylinositol 3-kinase activation by CXCR4. J Exp Med. 2010;207 (1):247-261.

16. Li M, Sun X, Ma L, et al. SDF-1/CXCR4 axis induces human dental pulp stem cell migration through FAK/PI3K/Akt and GSK3beta/beta-catenin pathways. Sci Rep. 2017;7:40161.

17. Reiske HR, Kao SC, Cary LA, Guan JL, Lai JF, Chen HC. Requirement of phosphatidylinositol 3-kinase in focal adhesion kinase-promoted cell migration. J Biol Chem. 1999;274(18):12361-12366.

18. Wang JF, Park IW, Groopman JE. Stromal cellderived factor-1alpha stimulates tyrosine phosphorylation of multiple focal adhesion proteins and induces migration of hematopoietic progenitor cells: roles of phosphoinositide- 3 kinase and protein kinase C. Blood. 2000;95(8):2505-2513.

19. Reinholdt L, Laursen MB, Schmitz A, et al. The CXCR4 antagonist plerixafor enhances the effect of rituximab in diffuse large B-cell lymphoma cell lines. Biomark Res. 2016; 4:12.

20. Beider K, Ribakovsky E, Abraham M, et al. Targeting the CD20 and CXCR4 pathways in non-hodgkin lymphoma with rituximab and high-affinity CXCR4 antagonist BKT140. Clin Cancer Res. 2013;19(13): 34953507

21. O'Callaghan $\mathrm{K}$, Lee L, Nguyen $\mathrm{N}$, et al. Targeting CXCR4 with cell-penetrating pepducins in lymphoma and lymphocytic leukemia. Blood. 2012;119(7):1717-1725.

22. Buchner M, Brantner P, Stickel N, et al. The microenvironment differentially impairs passive and active immunotherapy in chronic lymphocytic leukaemia - CXCR4 antagonists as potential adjuvants for monoclonal antibodies. Br J Haematol. 2010;151(2):167-178.

23. Recasens-Zorzo C, Cardesa-Salzmann T, Petazzi P, et al. Pharmacological modulation of CXCR4 cooperates with BET bromodomain inhibition in diffuse large B-cell lymphoma. Haematologica. 2019;104(4):778788.

24. Cao Y, Hunter ZR, Liu X, et al. The WHIMlike CXCR4(S338X) somatic mutation activates AKT and ERK, and promotes resistance to ibrutinib and other agents used in the treatment of Waldenstrom's Macroglobulinemia. Leukemia. 2015;29 (1):169-176.
25. Chapuy B, McKeown MR, Lin CY, et al. Discovery and characterization of superenhancer-associated dependencies in diffuse large $B$ cell lymphoma. Cancer Cell. 2013;24(6):777-790.

26. Currie KS, Kropf JE, Lee T, et al. Discovery of GS-9973, a selective and orally efficacious inhibitor of spleen tyrosine kinase. J Med Chem. 2014;57(9):3856-3873.

27. Sharman J, Hawkins M, Kolibaba K, et al. An open-label phase 2 trial of entospletinib (GS9973), a selective spleen tyrosine kinase inhibitor, in chronic lymphocytic leukemia. Blood. 2015;125(15):2336-2343.

28. Andorsky DJ, Kolibaba KS, Assouline S, et al. An open-label phase 2 trial of entospletinib in indolent non-Hodgkin lymphoma and mantle cell lymphoma. $\mathrm{Br} \mathrm{J}$ Haematol. 2019;184(2):215-222.

29. Kharas MG, Okabe R, Ganis JJ, et al. Constitutively active AKT depletes hematopoietic stem cells and induces leukemia in mice. Blood. 2010;115(7):14061415.

30. Ochiai K, Maienschein-Cline M, Mandal M, et al. A self-reinforcing regulatory network triggered by limiting IL-7 activates pre-BCR signaling and differentiation. Nat Immunol. 2012;13(3):300-307.

31. Tarantelli C, Gaudio E, Arribas AJ, et al. PQR309 is a novel dual PI3K/mTOR inhibitor with preclinical antitumor activity in lymphomas as a single agent and in combination therapy. Clin Cancer Res. 2018; 24(1):120-129.

32. Hunter ZR, Xu L, Yang G, et al. The genomic landscape of Waldenstrom macroglobulinemia is characterized by highly recurring MYD88 and WHIM-like CXCR4 mutations, and small somatic deletions associated with B-cell lymphomagenesis. Blood. 2014;123 (11):1637-1646.

33. Chen J, Xu-Monette ZY, Deng L, et al. Dysregulated CXCR4 expression promotes lymphoma cell survival and independently predicts disease progression in germinal center B-cell-like diffuse large B-cell lymphoma. Oncotarget. 2015;6(8):5597-5614.

34. Moreno MJ, Bosch R, Dieguez-Gonzalez R, et al. CXCR4 expression enhances diffuse large B cell lymphoma dissemination and decreases patient survival. J Pathol. 2015; 235(3):445-455.

35. Treon SP. How I treat Waldenstrom macroglobulinemia. Blood. 2015;126(6): 721732 .

36. Xu ZZ, Shen JK, Zhao SQ, Li JM. Clinical significance of chemokine receptor CXCR4 and mammalian target of rapamycin (mTOR) expression in patients with diffuse large Bcell lymphoma. Leuk Lymphoma. 2018;59(6): 1451-1460. 